Original Research Paper

\title{
Extracting Unique Personal Identification Number from Iris
}

\author{
${ }^{1}$ Nenad Nestorovic, ${ }^{1}$ P.W.C. Prasad, ${ }^{1}$ Abeer Alsadoon and ${ }^{2}$ Amr Elchouemi \\ ${ }^{I} S C M$, Charles Sturt University, Australia \\ ${ }^{2} I T$, Walden University, USA
}

Article history

Received: 24-02-2017

Revised: $16-05-2017$

Accepted: 07-07-2017

Corresponding Author:

P.W.C. Prasad

IT, Study Group Australia,

Australia

Email: cwithana@studygroup.com

\section{Introduction}

\section{Background}

Biometric authentication technology has been gaining much popularity in recent years. Personal identification based on biometrics finds application in number of sectors such as research centres, military complexes or international airports. Individuals are identified by assessing their physiological characteristics such as fingerprints, facial features, voice, eye structure or even DNA. Utilization of these unique biological identifiers makes biometrics authentication more secure identification method than

\begin{abstract}
The aim of this research is to propose an enhanced model that significantly reduces the noise in the iris templates and thus present an efficient and reliable iris recognition system. Accuracy of Iris recognition system significantly depends on reducing noise at every stage of recognition process especially at eye image acquisition and iris segmentation stages. The proposed Iris recognition model combines Canny edge detector and circular Hough transform which yields more rapid and accurate localization of Iris inner and outer boundaries. The eyelid and eyelashes occlusion is also dealt with by applying non maxima radial suppression technique. The segmented Iris area is then normalized and converted from annular shape to fixed-sized rectangular block. In this stage all iris templates converted from circular to rectangular shape will have standard dimensions thus enabling Iris recognition system to perform comparison between them. Fast Fourier Transform function is then applied to normalized Iris image. Input image is viewed as a signal that is converted from time to frequency domain in which the number of frequencies is equal to number of image pixels. The resulting matrix is consisted of real and imaginary part represented by complex numbers. The matrix is encoded according to the pre established encoding pattern that assigns certain sequence of binary numbers to each possible combination of complex numbers thus producing Iris code. In the matching phase the iris codes are compared and if the determined degree of dissimilarity between the two is below the pre-established threshold value than the codes belong to the same user. The proposed Iris recognition method, therefore, successfully treats eyelids and eyelashes noise issues and present the efficient set of algorithms whose performance is superior compared to other existing methods.
\end{abstract}

Keywords: Image Acquisition, Eyelashes Detection, Edge Detection, Iris Normalization, Iris Template, Moment Invariants traditional passwords, for instance. It is mainly because these identifiers, unlike passwords, cannot be stolen, lost or forgotten. Iris recognition, likewise, deals with identifying users by scanning and analysing the Iris pattern extracted from their eye images (Fig. 1). Iris biometrics in particular is widely adopted form of biometric authentication because of its distinguishing properties. These properties include stability and rich texture. Iris is a stable biometrics, meaning that, unlike face or voice, it does not change during the individual's life time. Due to Iris unique set of significant features possibility of finding two individuals with identical Iris patterns is almost zero. 


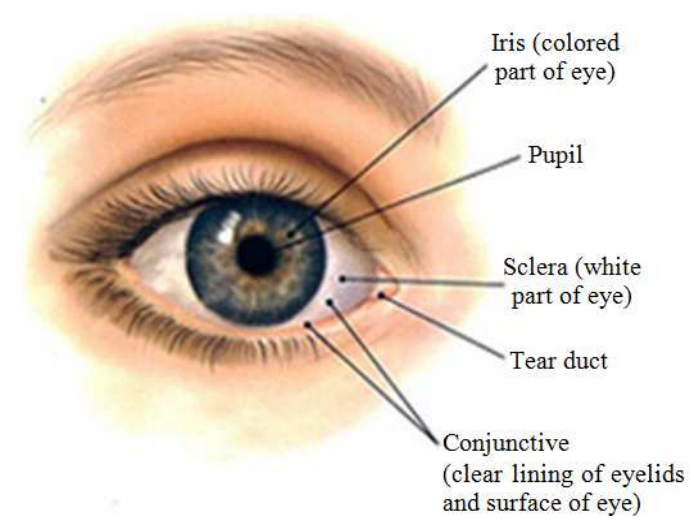

Fig. 1. Eye structure

The Iris biometric system has two security stages: The enrolment stage and the release stage. During the enrolment stage, samples of Iris biometric are collected. The samples are then measured and the data is stored in a database. In the release stage, sample data is taken again from a subject and compared to the existing data stored in the database. If two samples are matched the identification was successful.

Once the Iris sample is obtained from a user the process of iris features extraction and iris code generation, takes place. This process is consisted of several steps. Eye image collection (acquisition) involves scan of a human eye, performed by a capturing device such as infrared camera. Iris localizing/segmenting refer to defining iris inner and outer boundaries and segmenting Iris from the eye image. In the Iris normalization step all the Iris templates, which may vary in size, are converted into the standard dimensions thus allowing easier further processing. Normalization also compensates certain inconsistencies such as distance and angle of the eye with respect to the camera, or pupil size variation due to the different illumination. The iris feature extraction step is a process where unique iris features, such as stripes, freckles, coronas, rings etc. are converted to a set of mathematical parameters. These parameters are than encoded which enables comparisons between iris codes in the identification stage.

\section{Issues}

Despite many advantages over other authentication methods, the Iris biometrics likewise is not speared from certain drawbacks. The major issue is the occurrence of noise in biometric samples. Noise occurs when there is a difference between the same biometric measurements. The number of factors can contribute to this problem. The measurements can be different due to injuries, sickness, aging, etc. Also many technical factors such as ambient lighting reflections change in the angle of the user's head during image capturing process or occlusions like eyelashes or eyelids or even the contact lenses can cause noise in the input images. Due to these factors the iris codes obtained from the same subject will have certain degree of dissimilarity. If that degree is high the false rejection rate will be increased as well. Since the process of extracting iris code from iris image involves several complex algorithms it is important that each of them contribute to minimum level of noise. Each stage at extraction process can contribute to noise occurrence: Capturing eye image with inadequate lightning, improper segmentation etc. Many of the existing methods do not offer solution for seemingly irrelevant issues such as eyelid and eyelash occlusion.

The problem of noise in samples if not addressed adequately can lead to increase of system errors such as false acceptance error where the system grants access to an unauthorized person and therefore there is a need for a system that is backed up with algorithms that will reduce noise in samples to minimum.

\section{Aims}

The project will research the proven algorithms employed to extract the iris code from iris image that are proposed in the available literature. While studying those algorithms a number of parameters will be identified that will help in determining which of them can be included in the proposing hybrid solution and why. This will be done by analysing advantages and drawbacks of each particular algorithm involved in the process. The algorithms can possibly be tested by using adequate software such as Matlab where for many of these algorithms the corresponding code can be developed or used and modified already developed ones that are available. The eye images from Chinese Academy of Sciences (CASIA) database can be used for that purpose. Based on the conducted studies a potential new approach that offers improved iris extraction process will be developed. The approach will be comprised of algorithms with strongest parameters where each algorithm corresponds to particular stage in iris feature extracting process (segmentation, normalization etc). Step-by-step solution will be presented so that such method can be potentially adopted and implemented.

\section{Literature Review}

Since the first automated Iris recognition concept was proposed by Flom and Safir (1987), many researchers have been introducing their models based on diversity of techniques and innovative approaches. John Daugman's work based on integro-differential operator used to localize Iris boundaries and Gabor filter used for Iris feature extraction for years has been inspiration and role model for many researchers. In general quite a few researchers followed the same main idea where the Iris recognition system was comprised of five major steps 
namely image acquisition, Iris segmentation, Iris normalization, feature extraction and matching.

In one of the recently proposed methods for iris recognition system, some popular techniques such as Circular and Linear Hough transform algorithm are used for Iris segmentation whereas Daugman's rubber sheet model is used in normalization stage. Several preprocesing steps are included as well to enhance image contrast. This particular set of techniques yields relatively good performance. However experimental results indicate that false rejection rate is fairly higher (around 2\%) compare to Daugman's approach where FFR is only $0.01 \%$ (Mahlouji and Noruzi, 2012).

Another approach includes Canny edge detector to determine iris boundaries in segmentation phase. The advantages of such technique are low cost and suitability in working with non-ideal perioucular images. Along with few other widely adopted algorithms, this approach also contains a novel technique for feature extraction. The technique is based on edge detection that changes image into binary format. The image is processed then in both radial and circular ways thus generating iris code (Rakesh and Khogre, 2012). However, due to the lack of experimental results not much can be done to prove researchers claims about superiority of the Iris recognition model. The next reviewed journal, that proposes a fast wavelet transform method applied in feature extraction stage, describes advanced version of wavelet transform technique that is based on computing the gradient direction of the Iris features. Resulting pixels are then encoded by following the pre-established encoding pattern (Oluwakemi et al., 2014). This method, similarly to others in reviewed papers, does not entirely address the noise caused by eyelid and eyelash occlusion, as well as pupils variation issues and light reflection that may occur as a consequence of improper image capturing. Many proposed Iris recognition methods, yet, are based on Haar wavelet algorithm which is computationally inexpensive, fast and accurate and can achieve average recognition rate over $95 \%$. Haar function is used for feature extraction stage and it can be described as a form of compression the employs averaging and differencing operations thus creating coefficients that will be encoded in the letter stage. According to the conduced experiments the entire method has high accuracy and low complexity rate (Srivastava, 2013). The disadvantage, in this case, is reduced processing speed since some additional steps required extended processing time. One of those steps was use of Gaussian mask as a masking technique. Some researchers did not fully follow the Daugman's path which is founded on five well know steps. They rather came up with their innovative approaches that incorporated not so widely adopted and used techniques especially in the Iris feature extraction section. One of such approach is lamstar neural network model. Here, each column in Iris template is considered to be subwords. These subwords are used for setting the weights of the SOM modules of the LAMSTAR. The SOM-module is based on "Winner takes all" neurons, the winning neuron has an output of 1 , while all other neurons in that SOM module have zero output (Homayon, 2015). Many other researchers are introducing fairly novel techniques for Iris feature extraction such as, for instance, the scale-space filtering technique. This technique is based on the variations in the directional properties of image brightness. The advantage of this approach is the ability to fully preserve the original information while in the same time the effect of the noise is minimized. Encoding is conducted by following pattern: Pixel with positive second derivatives is encoded with 1 while 0 is given to negative second derivatives (Yang, 2013). Despite the relatively high efficiency and reliability, the testing has indicated significant rate of false rejection error. Another appealing proposal in regards to the iris feature extraction is a model called cumulative sum based gray change analysis. In this algorithm the changes of gray values of iris patterns are analysed. Upward slope of cumulative sums indicates that iris pattern will change from darkness to brightness; downward slope of cumulative sums indicates the opposite change of upward slope (Taherian and Aliyari, 2013). This method, along with some other commonly used algorithms, compose an efficient technique that has high rate of accuracy. The drawback, however, is lack of interest to address for potential eyelid or eyelash occlusion issues. In one of the latest proposal Iris segmentation is performed with Circular Hough transform, normalized Iris image is then enhanced using histogram equalizing to enhance image contrast and the feature extraction is performed using 1D Gabor filter. The testings have shown that Circular Hough transform could be computationally intensive, Gabor filter has a high complexity rate and Iris enhancement step does not contribute to system performance.

\section{Proposed Model}

\section{Methodology}

The Iris biometric system is generally comprised of hardware for capturing eye image and software for performing Iris recognition. In this model instead of manually capturing eye images from the subjects, already existing CASIA eye image database is used. The data base is available for downloading from CASIA web page. In regards to the software that performs Iris recognition an improved code that can run on MATLAB R2015a platform has been developed. This code has number of functions that are exact representation of proposed model that includes all corresponding steps from the Iris segmentation to the matching. The proposed model is given in Fig. 2. 


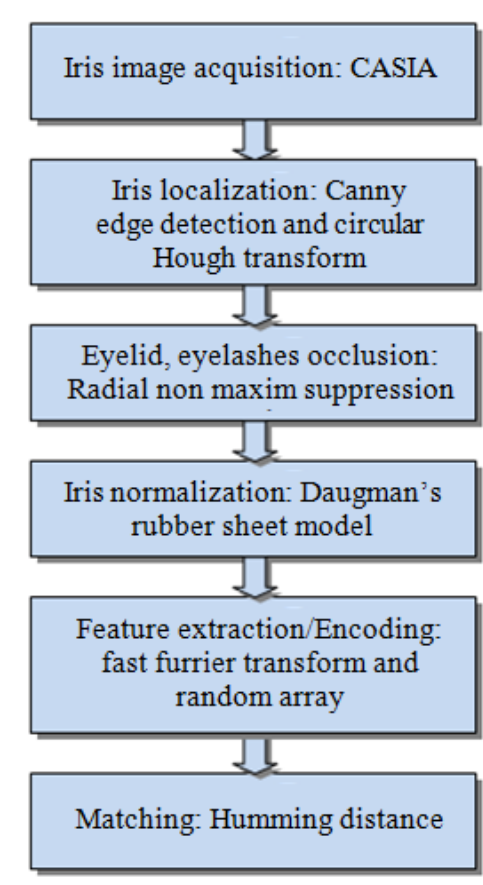

Fig. 2. Flow chart of proposed model

\section{Iris Image Acquisition}

In this stage the eye image is captured from the subject using capturing device. Digital camera with good resolution or infra red sensor can be utilized. Completing this step appropriately is of high importance because a clear image normally does not contain much noise that needs to be eliminated in the later stages. For this research purposes the Chinese Academy of Science Institute of Automation (CASIA) eye image database has been used.

\section{Iris Localization}

Iris localization refers to identifying Iris inner and outer (Iris-pupil and Iris-sclera) boundaries. For that purpose a combination of Circular Hough Transform algorithm and Canny Edge detector has been used. The purpose of the Circular Hough Transform is to detect circles in imperfect image inputs. The circle candidates are produced by "voting" in the Hough parameter space and then select the local maxima in a so-called accumulator matrix. Due to "Brute-force" approach, where Circular Hough Transform analyses every potential circle in the input image, the technique can be computationally intensive. For that reason Canny Edge detector is employed first to create an edge map in the input eye image which then hugely helps Hough Transform to more rapidly and more accurately determine the Iris boundaries. First the Iris-sclera boundary is defined and then the system within that circle searches for Iris-pupil boundary.

\section{Radial Non Maxim Suppression}

This technique is used for eyelid and eyelashes detection and their removal since they represent noisy information in the input eye image. The image gradient direction is scanned and if the pixels are not within of the local maxima scope those pixels are set to zero. In the other words the suppressing of all image information that is not part of local maxima is performed. The following is the algorithm of the Radial non maxim suppression.

Input: Im (the input eye image) and $(\mathrm{xc}, \mathrm{yc})($ the approximate centre point of the edge).

Output: Rm (the resulting eye image after applying radial non maxima Suppression).

1. for all points $\mathrm{Pj}$ of the input eye image $\mathrm{A}$ do

2. find out the radial direction of point $\mathrm{Pj}$ meaning the direction from approximate centre point to $\mathrm{Pj}$.

3. find out two points $\mathrm{P} 1$ and $\mathrm{P} 2$ in the 8 neighbouring pixels of $\mathrm{Pj}$ along the radial direction.

4. if $A(P j)>A(P 1)$ and $A(P j)>A(P 2)$ then $m(P j)=$ $\mathrm{A}(\mathrm{Pj})$

5. else $m(P j)=0$

6 . end if

7. end for

8. return $\mathrm{Rm}$.

3.5 Iris Normalization

Normalization prepares a segmented iris image for the feature extraction process.

Because extracted iris has different size and value, patterns should be translated into fixed size dimensions to fit a classifier and that is achieved with normalization. Captured iris images may have different distance and angular position with respect to the camera. Also, pupil size varies when exposed to different amount of light. A proper normalization technique transforms the iris image to compensate these variations.

Most normalization approaches based on Cartesian to polar transformation unwrap the annular iris texture into a fixed-size rectangular block. The Cartesian to polar transform is defined as:

$$
\begin{aligned}
& x(1 / 2 ; \mu)=(1 ; 1 / 2) £ x p(\mu)+1 / 2 £ x i(\mu) ; \\
& y(1 / 2 ; \mu)=\left(1 ; \frac{1}{2}\right) \mathfrak{f} y p(\mu)+1 / 2 £ y i(\mu) ;
\end{aligned}
$$

where:

$$
\begin{aligned}
& x p(\mu)=x p 0(\mu)+r p £ \cos (\mu) ; \\
& y p(\mu)=y p 0(\mu)+r p £ \sin (\mu) ; \\
& x i(\mu)=x i 0(\mu)+r i £ \cos (\mu) ; \\
& y i(\mu)=y i 0(\mu)+r i £ \sin (\mu):
\end{aligned}
$$




\section{Iris Feature Extraction}

Once the Iris normalization is completed the significant features must be extracted. This Iris recognition method involves Fast Furrier Transform function to perform Iris feature extraction. FFT is a simple function that converts signal from time to frequency domain and vice versa. The normalized Iris image can be viewed as an input signal. The number of frequencies will be equal to the number of image pixels. The resulting signal will have real and imaginary part. The advantage of working in the frequency domain is more accurate and simpler operation performing. Since the signal is decomposed into smaller components, analysing and comparison is much easier. The algorithm takes column by column from the normalized iris image and convolves it with FFT function. The resulting vector will contain real and imaginary part represented by complex numbers. Once the entire Iris image is processed resulting matrix will be encoded according to the pre-established encoding pattern. The experimental results has shown that FFT function has certain advantages compare to some other techniques such as Gabor filter, for instance. Gabor filter, in particular, is a complex operator that has higher componential complexity compare to FFT. Also Gabor filter overall accuracy is slightly lower than with FFT.

\section{Encoding}

Each value in the resulting vector, obtained by convolving image column and FFT function, will have real and imaginary part. Both parts can be either positive or negative. Therefore there are four possible combinations for each complex number that will be encoded. For example if the resulting value contains positive real and positive imaginary part the corresponding code will be 00 . If the value contains positive real and negative imaginary part the corresponding code will be 01 and so on. Once the entire resulting matrix is encoded the Iris code is obtained and it is comprised of sequence of binary numbers. Such code is ready to be compared to other codes stored in a data base.

\section{Matching}

When a user wishes to be identified his/her eye image is captured and converted to Iris code which is then compared to other Iris codes stored in a database. In biometrics systems there are two type of authentication. Identification mode refers to one-to-many templates comparison where as in the verification mode one-to-one comparison is performed. When comparing Iris codes two types of error may occur in the biometric system: False Acceptance Rate (FAR) and False Rejection Rate (FRR). FAR is a percentage of non-authorized individuals who were accepted by the system. FRR is a percentage of valid users rejected by the system.
In the proposed model Hamming Distance (HD) algorithm is used for Iris code comparison. Hamming distance simply counts the number of different bits between two equally length binary numbers thus determining the degree of dissimilarity between them. Due to many factors eye images captured from the same user will always have certain degree of dissimilarity. For that reason it is necessary to establish threshold value for acceptable level of the dissimilarity. The testings show that this value should be around 0.35 . If the HD is below that value the codes are match otherwise they belong to different users.

\section{Results}

Figure 3 a shows the input eye images taken from the CASIA database. Once the Images are selected the system determines whether or not they belong to the same user. Figure $3 \mathrm{~b}$ shows the segmented iris performed by Canny edge detector and circular Hough transform. Figure $4 \mathrm{~b}$ demonstrates marked eyelid and eyelashes that occlude Iris. Figure 5 denotes unwrapped normalized Iris images. Figure 6 shows the iris template. Table 1 demonstrates Hamming distance values when randomly chosen eye images from the same user are compared. The testing was conducted firstly applying Gabor filter in the feature extraction stage and then when Fast Fourier Transform is performed in the same stage. The experimental results show that Hamming distance has a slightly lower value in the case of proposed model that uses FFT. This indicates that the proposed model operates more precisely then existing solutions. In particular, due to this fact the threshold value for $\mathrm{HD}$ can be lowered from 0.35 to 0.325 according to the experimental results. In this case the recognition rate with model based on Gabor filter would drop from $93 \%$ to only $80 \%$ where as the recognition rate in proposed model would remain $100 \%$. Table 2 given the comparison between randomly chosen eye images from the different users.

The Fig. 7 shows testing results in regards to recognition rate for both existing solution based on Gabor filter feature extraction and proposed method based on FFT. The threshold value for Hamming Distance (HD) varies from 0 to 0.5 . The existing solution achieves $100 \%$ recognition rate when $\mathrm{HD}$ is 0.375 where as proposed model achieves $100 \%$ accuracy when HD is 0.325 .

Similarly the Fig. 8 compares false rejection rate with different threshold values for both existing and proposed method. With existing solution FRR remains zero when HD is equal or below 0.375 where as in proposed model FRR is zero as long as the HD is equal or below 0.325 .

Figure 9 shows that in proposed model recognition rate will be $100 \%$ and FRR and FAR $0 \%$ when HD value is between 0.325 and 0.375 . 


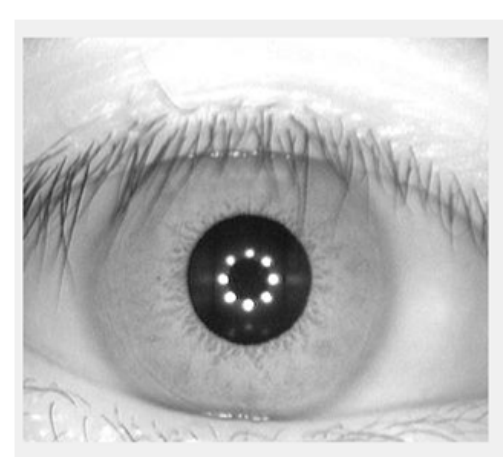

(a)

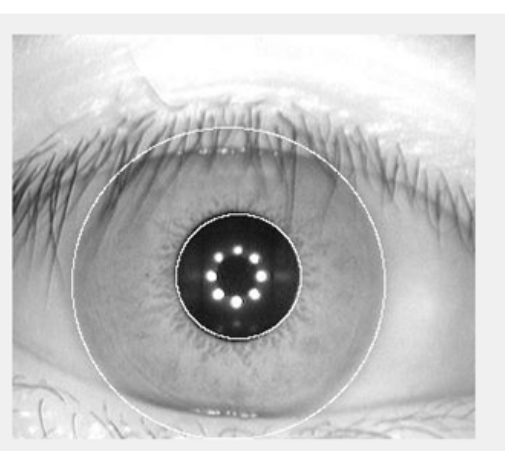

(b)

Fig. 3. (a) Original eye image (b) Iris inner and outer boundaries localized

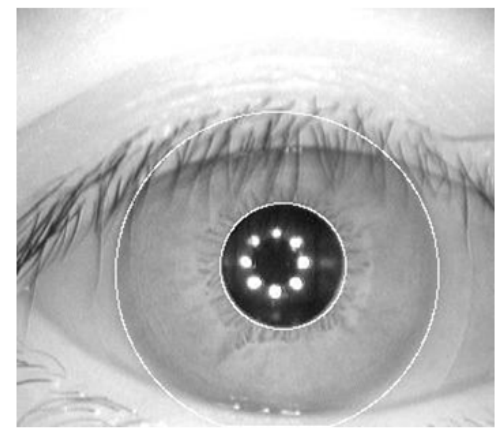

(a)

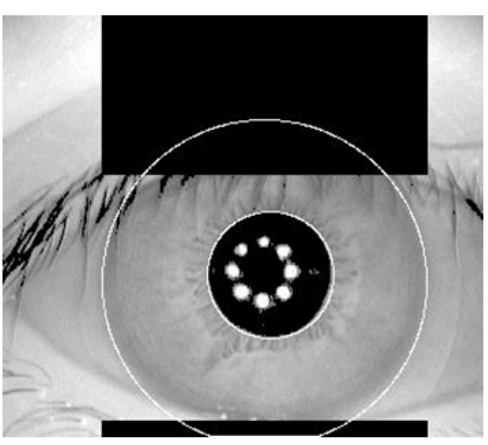

(b)

Fig. 4. (a) Segmented Iris (b) Eyelids and eyelashes suppressed
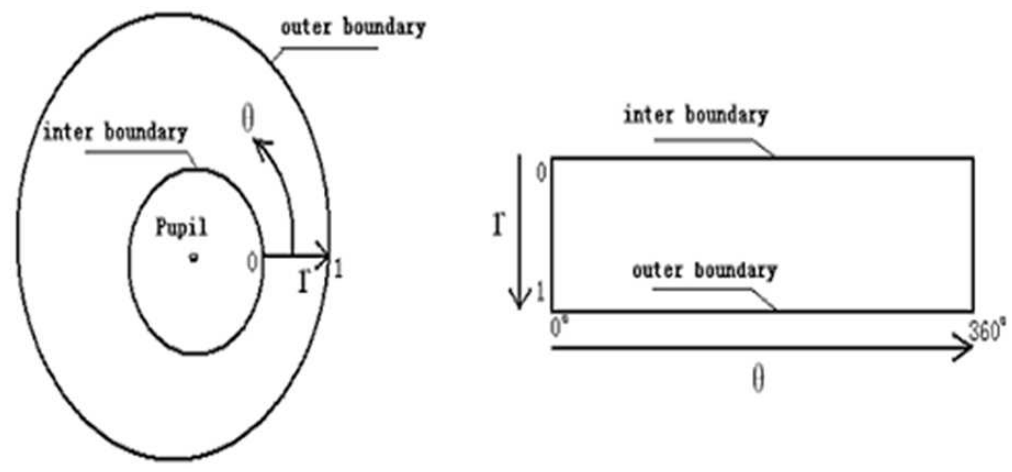

Fig. 5. Transforming polar to Cartesian coordinates

\begin{tabular}{|l|c|}
\hline \multicolumn{1}{|c|}{ Complex number } & Corresponding code \\
\hline Positive real part, positive imaginary part & 00 \\
\hline Positive real part, negative imaginary part & 01 \\
\hline Negative real part, negative imaginary part & 10 \\
\hline Negative real part, negative imaginary part & 11 \\
\hline
\end{tabular}

TableXX: Encoding pattern for

\section{Iris Template}

01000010111101010010101111010100101011010101010100 10100111000111101110100010011000110110110001011110

Fig. 6. Example of iris template 


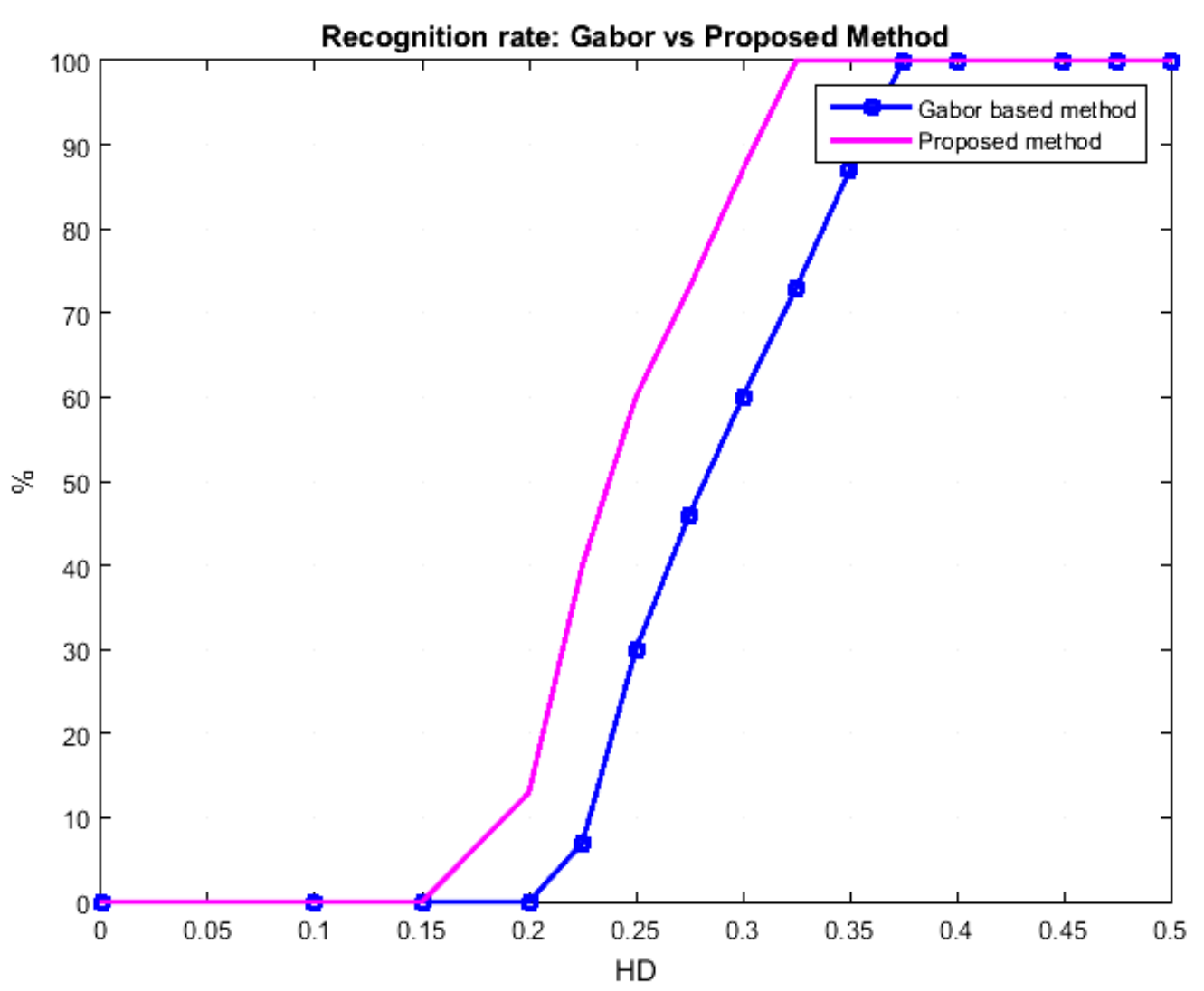

Fig. 7. Comparison of recognition rate between existing and proposed method

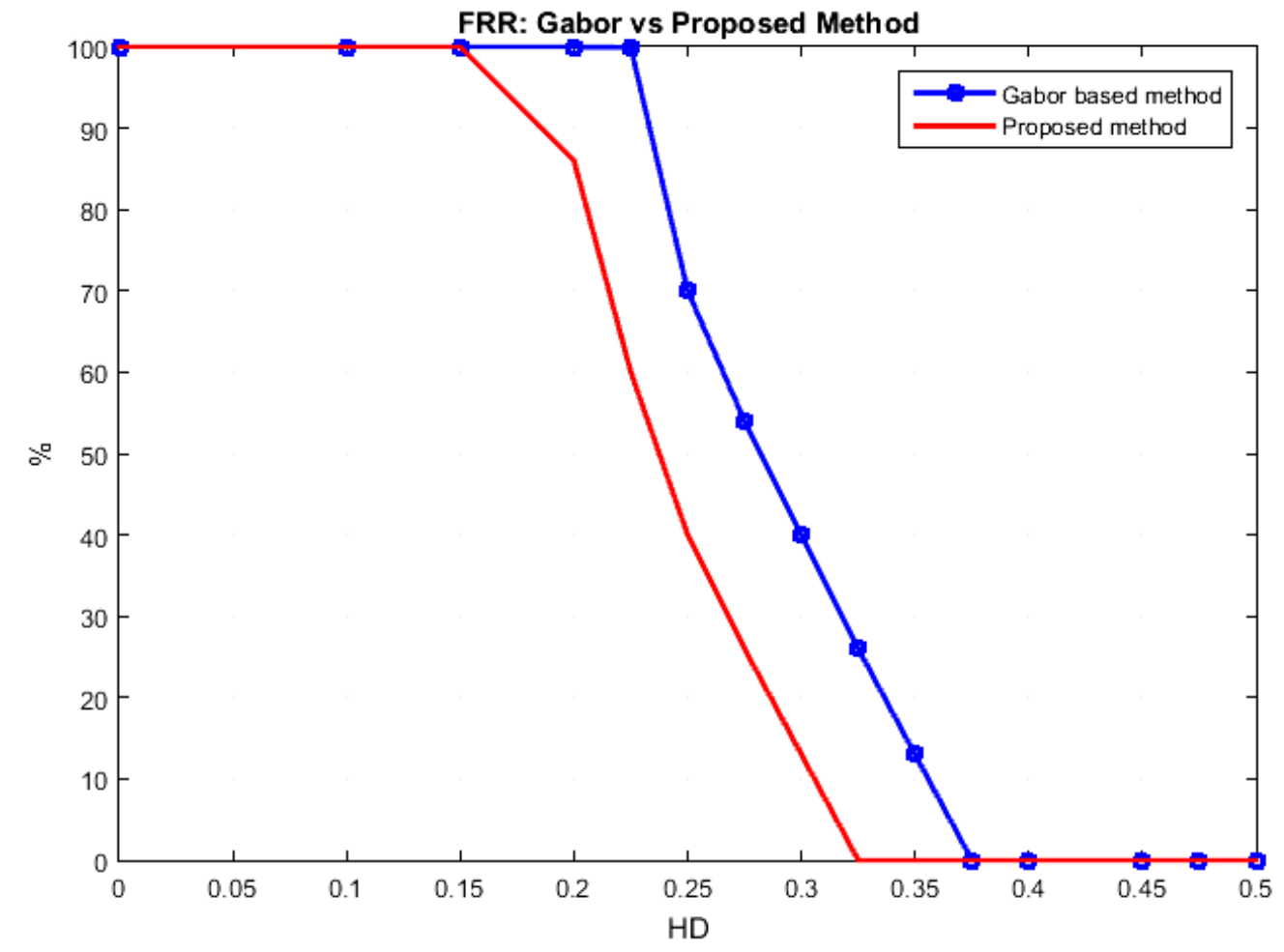

Fig. 8. Comparison of FRR rate between existing and proposed method 


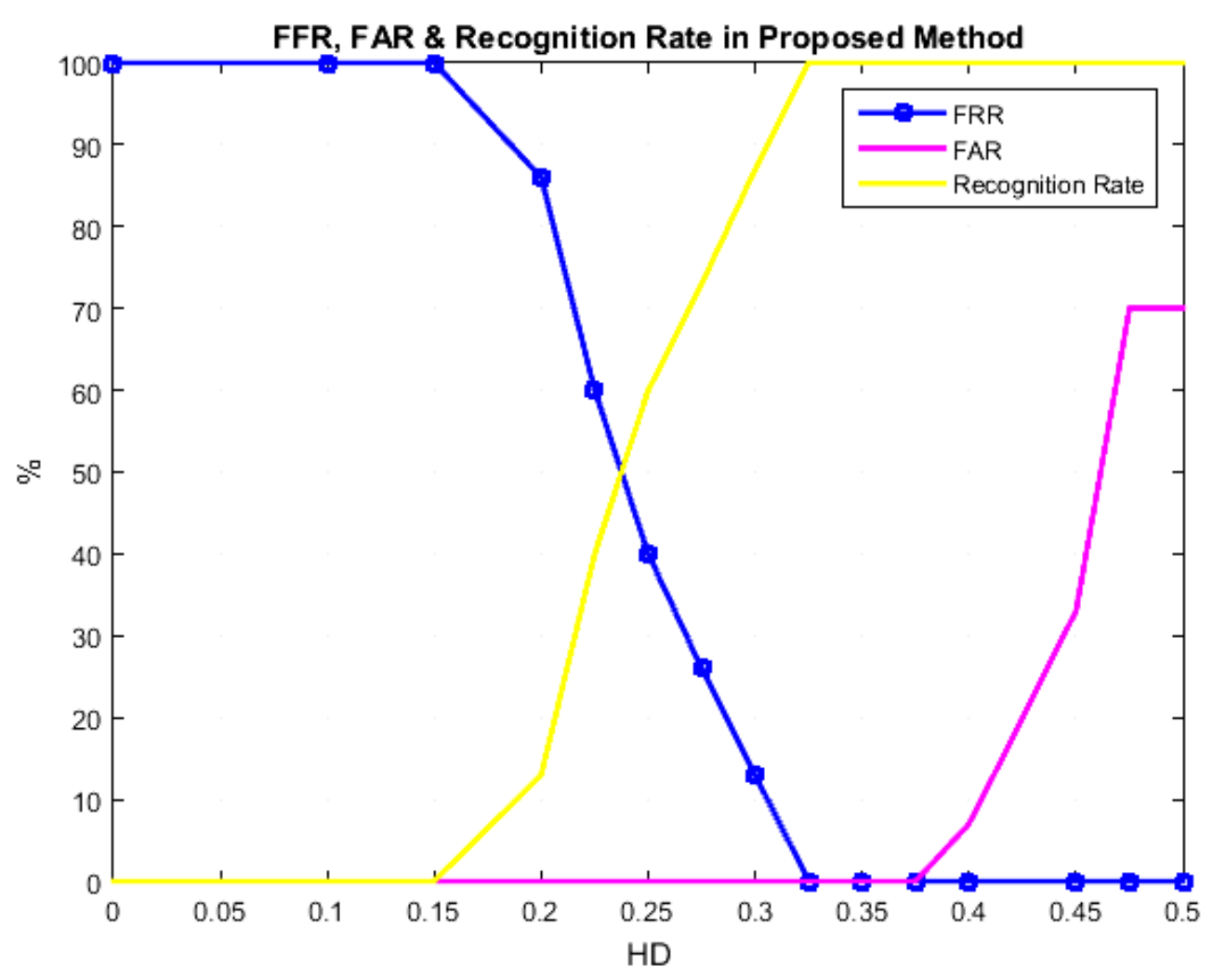

Fig. 9. Percentage of FRR, FAR and recognition rate in proposed method

Table 1. Comparison between randomly chosen eye images from the same user

\begin{tabular}{|c|c|c|c|c|c|c|}
\hline \multirow[b]{2}{*}{ User no. } & \multicolumn{3}{|l|}{ With Gabor filter } & \multicolumn{3}{|c|}{ With Proposed Method } \\
\hline & 1. Eye image no & 2. Eye image no & $\mathrm{HD}$ & 1. Eye image no & 2. Eye image no & HD \\
\hline 001 & 1 & 5 & 0.2717 & 1 & 5 & 0.2181 \\
\hline 002 & 3 & 7 & 0.3643 & 3 & 7 & 0.3225 \\
\hline 003 & 2 & 6 & 0.2357 & 2 & 6 & 0.2221 \\
\hline 004 & 5 & 7 & 0.2458 & 5 & 7 & 0.2404 \\
\hline 005 & 2 & 4 & 0.3377 & 2 & 4 & 0.2053 \\
\hline 006 & 3 & 6 & 0.2798 & 3 & 6 & 0.1909 \\
\hline 007 & 5 & 9 & 0.3254 & 5 & 9 & 0.2739 \\
\hline 008 & 2 & 4 & 0.3009 & 2 & 4 & 0.2266 \\
\hline 009 & 1 & 3 & 0.2309 & 1 & 3 & 0.2766 \\
\hline 010 & 1 & 3 & 0.2085 & 1 & 3 & 0.1789 \\
\hline 011 & 4 & 8 & 0.2818 & 4 & 8 & 0.2395 \\
\hline 012 & 1 & 6 & 0.2724 & 1 & 5 & 0.3086 \\
\hline 0131 & 3 & 6 & 0.3193 & 3 & 6 & 0.2642 \\
\hline 0141 & 2 & 4 & 0.3464 & 2 & 4 & 0.2983 \\
\hline 015 & 2 & 3 & 0.2270 & 2 & 3 & 0.2205 \\
\hline \multicolumn{2}{|c|}{ Recognition Rate: } & $93 \%$ & \multicolumn{2}{|c|}{ Recognition Rate: } & $100 \%$ & \\
\hline
\end{tabular}

\section{Evaluation of the Model}

According to the testing results the proposed model clearly has better overall performance than existing solution. The following is highlight of major differences between the two models. The existing model utilizes circular Hough transform that determines the Iris boundaries while in the proposed model the Canny edge detector is added to assist Hough transform in performing that task. Circular Hough transform if operates alone it could be a computationally intensive because every potential circle in the input image will be analysed. With assistance of Canny edge detector which creates edge map finding Iris inner and outer boundaries can be performed much faster and with higher accuracy. 
Table 2. Comparison between randomly chosen eye images from the different users

\begin{tabular}{|c|c|c|c|c|c|c|}
\hline & \multicolumn{3}{|l|}{ With Gabor filter } & \multicolumn{3}{|l|}{ With Proposed Method } \\
\hline & 1. User no/image no: & 2. User no/image no: & HD & 1. User no/image no: & 2. User no/image no: & HD \\
\hline 1 & $1 / 1$ & $2 / 2$ & 0.4533 & $1 / 1$ & $2 / 2$ & 0.4615 \\
\hline 2 & $2 / 2$ & $3 / 3$ & 0.4609 & $2 / 2$ & $3 / 3$ & 0.5455 \\
\hline 3 & $3 / 3$ & $4 / 4$ & 0.4670 & $3 / 3$ & $4 / 4$ & 0.4650 \\
\hline 4 & $4 / 4$ & $5 / 5$ & 0.4569 & $4 / 4$ & $5 / 5$ & 0.4353 \\
\hline 5 & $5 / 5$ & $6 / 6$ & 0.4461 & $5 / 5$ & $6 / 6$ & 0.3885 \\
\hline 6 & $6 / 1$ & $7 / 1$ & 0.4795 & $6 / 1$ & $7 / 1$ & 0.4519 \\
\hline 7 & $7 / 7$ & $1 / 1$ & 0.4777 & $7 / 7$ & $1 / 1$ & 0.4610 \\
\hline 8 & $1 / 2$ & $3 / 4$ & 0.4829 & $1 / 2$ & $3 / 4$ & 0.5070 \\
\hline 9 & $5 / 6$ & $7 / 8$ & 0.4659 & $5 / 6$ & $7 / 8$ & 0.4419 \\
\hline 10 & $2 / 8$ & $7 / 6$ & 0.4917 & $2 / 8$ & $7 / 6$ & 0.5365 \\
\hline \multicolumn{2}{|c|}{ Recognition Rate: } & $100 \%$ & \multicolumn{2}{|c|}{ Recognition Rate: } & $100 \%$ & \\
\hline
\end{tabular}

The second key difference is enhancement of normalized Iris image performed in the existing model. The testings, however, show that this step does not contribute to the improvement in the overall system performance and it is simply unnecessary. Therefore, this step is omitted in the proposed solution. The last key difference is employment of different techniques in the feature extraction phase. While existing model suggests use of 1D Gabor filter, in the proposed model Fast Fourier Transform function is applied to extract significant Iris features. With second option the system complexity is slightly reduced and results indicate higher recognition rate. Furthermore, the improved method unlike many previously proposed models, deals with eyelid and eyelash occlusion that is consider to be noise in the input eye image. Finally, when two models are directly compared using MATLAB software it is obvious that the presented Iris recognition system represents noticeably improved version of the existing model.

\section{Conclusion}

Iris recognition is the most reliable authentication biometric techniques because of uniqueness of iris features and anatomy of that particular part of human eye. The Iris authentication process includes enrolment and identification/verification stages. In both stages the individual's eye is scanned and the eye image is further processed through several steps. The result of the processing is the iris template. Iris templates are then mutually compared database so that the identity of the subject can be established.

In every captured eye image the eyelid and eyelash occlusion can represent noisy information that is undesirable in the Iris templates. In order to adequately extract significant features from Iris image, normalized Iris must be minimally affected by these types of noise. The proposed scheme successfully deals with this issues while in the same time accurately identifies Iris inner and outer boundaries. The proposed method also has lower complexity rate due to deployment of FFT which is a simple feature extraction function. The degree of difference between two iris codes determines whether or not the codes are from the same user.

The presented solution represents improved method for Iris feature extraction and possesses significantly better characteristics than other existing methods.

\section{Acknowledgment}

We are grateful to Mrs. Angelika Maag for proof reading and making corrections to this article. Without her support, it would have not been possible to submit this in current form.

\section{Author's Contributions}

Nenad Nestorovic: Completed this research project on Extracting Unique Personal Identification Number from Iris as part of his MIT degree program. $\mathrm{He}$ has investigated the issues with personal identification numbers and proposed a new method to identify it using the Iris.

P.W.C. Prasad: Supervised/worked closely with NNenad during the analysis, design and experiment phases.

Abeer Alsadoon: Worked on the setup of the experiments and gave important suggestions on design of experiments.

Amr Elchouemi: Made important revisions to most sections of the paper. Give the final review and approval for the manuscript to be submitted.

\section{Ethics}

This article is original and contains unpublished material. The corresponding author confirms that all of the other authors have read and approved the manuscript and no ethical issues involved. 


\section{References}

Flom, L. and A. Safir, 1987. Iris recognition system. US Patent no. 4641349.

Homayon, S., 2015. Iris recognition for personal identification using lamstar neural network. Int. J. Comput. Sci. Inform. Technol., 7: 1-8.

Mahlouji, M. and A. Noruzi, 2012. Human iris segmentation for iris recognition in unconstrained environments. Int. J. Comput. Sci., 9: 149-155.

Oluwakemi, A.C., J.S. Sadiku, K.S. Adewole and G.J. Rasheed, 2014. Iris feature extraction for personal identification using Fast Wavelet Transform (FWT). Int. J. Applied Inform. Syst., 6: 1-6.
Rakesh, T. and M.G. Khogre, 2012. Iris bio-metric feature for personal identification. Int. J. Eng. Res. Applic., 2: 2862-2867.

Srivastava, H., 2013. Personal identification using iris recognition system, a review. Int. J. Eng. Res. Applic., 3: 449-453.

Taherian, A. and M.S. Aliyari, 2013. Noise resistant identification of human iris patterns using fuzzy ARTMAP neural network. Int. J. Security Applic., 7: 105-118.

Yang, W.S., 2013. Personal identification using iris recognition. Department of Electronics and Electrical Engineering, Hongik University SejongRho 2639, Sejong, Korea. 